perifèria

Número 2, mayo 2005

www.periferia.name

\title{
Afinidades en la formación de las redes personales de los misioneros de la I glesia SUD en Barcelona
}

Margarita Lozano - U.A.B.- Estudiante de doctorado en Antropología Social ${ }^{1}$

Lo que pretendo demostrar en el presente artículo es que un grupo con valores marcadamente religiosos (como es el grupo de los mormones) y con un alto grado de personas procedentes de diferentes países, establece su identidad mediante una fuerte cohesión interna en una sociedad que oscila mayoritariamente entre el laicismo y el catolicismo. Esta cohesión interna, según las observaciones de campo, no indica homogeneidad, sino que existen diferencias de género, edad y culturales. He de advertir que los resultados presentados del sondeo realizado no se pueden extrapolar al resto de la organización religiosa de la Iglesia SUD $^{2}$ en Barcelona, por el escaso número de personas encuestadas ( 8 voluntarios) y por la homogeneidad del grupo que participó en el mismo.

La elección de la I glesia SUD ${ }^{3}$ de Barcelona viene motivada por la curiosidad que me despertaba el hecho de ver jóvenes varones no autóctonos que rondan los 20 años en el metro con una estética muy determinada que consistía en traje y carpeta de comercial. Esta estética me llamaba la atención por la fuerte diferencia que mantiene con la de jóvenes de su edad.

Una vez que empecé la observación de campo pude ver que dentro de la organización religiosa había una infraestructura dedicada a la racionalización del tiempo libre ${ }^{4}$, a la redistribución de bienes económicos, entre los que se encuentra la ayuda a los miembros con menos recursos, un

\footnotetext{
${ }^{1}$ Enviar correspondencia a: margarita.lozano@campus.uab.es

${ }^{2}$ Acrónimo de Santos de los Últimos Días.

3 Popularmente conocidos como mormones. Utilizo el término Iglesia SUD (Iglesia de los Santos de los Últimos Días) porque es así como prefieren ser conocidos.

${ }^{4}$ La organización del tiempo libre cuenta con grupos de estudio de la Biblia y los textos sagrados de la Iglesia SUD, sesiones de cine, excursiones, grupos de teatro y otros.
} 


\section{perifèria \\ Número 2, mayo 2005 \\ www. periferia.name}

fuerte sentimiento de comunidad, a pesar del elevado número de personas de distinta procedencia y una fuerte división de las funciones establecida por sexo, rol y status. Los discursos establecidos, al igual que la participación en los ritos tienden a naturalizar la diferencia dentro de la organización. La racionalización del tiempo libre parece destinada a la potenciación del sentimiento de comunidad utilizando todo el aparato sociocultural disponible y así según Horowitz, Irving, L. (1963:320) este aparato es dónde se produce y distribuye el arte, conocimiento y creencias.

La duda que empezó a surgir cuando intenté establecer los límites del sentimiento de pertenecer a una comunidad y las relaciones personales que se establecen dentro de la misma fue que no sabía si bastaba el hecho de pertenecer a la misma religión a la hora de establecer las relaciones personales y grupos de influencia o si las otras variables como la procedencia, el status socio-económico, el rol dentro de la organización o el nivel educativo pesaban más que la religión.

La I glesia SUD en Barcelona cuenta con aproximadamente 5.800 miembros en el área metropolitana de Barcelona. La división administrativa de la misión de Barcelona es la siguiente: hay dos estacas, división administrativa en que se divide una misión en la Iglesia SUD, la de Barcelona que a su vez se subdivide en 3 unidades con 2.800 miembros y la de L'Hospitalet, que comprende unos 3.000. El actual grupo cuenta con un elevado número de personas de distintas nacionalidades, siendo los latinoamericanos el grupo más numeroso.

Uno de los principales problemas que me he encontrado es la escasa bibliografía publicada sobre la Iglesia SUD fuera de sus publicaciones oficiales.

\section{Hipótesis}

(1) La elaboración de la hipótesis de trabajo ha estado basada en el diagnóstico que Herbert Marcuse hace de la organización burocrática. 


\section{perifèria \\ Número 2, mayo 2005 \\ www.periferia.name}

Los discursos establecidos dentro de la organización tienden a sancionar las diferencias de rol y estatus basadas en una división del trabajo por género y de acceso al saber. Este tipo de estatus formalmente establecido es racional y quienes están al frente deben su posición gracias al grado de confianza recibida, exigencia de disciplina y estabilidad además de contar con un saber fijo, calculable y distribuido por un equipo de expertos ${ }^{5}$.

De esta hipótesis se desprende que (2) las posiciones más centrales dentro de una sociored son los que corresponden con estas expectativas, y en la red ocupará la posición central el que posea estas características.

Otra hipótesis que se deduce de la primera es que (3) "el equipo de expertos" no necesariamente puede ocupar posiciones centrales sino que parte del equipo de expertos que distribuye la información a escala más básicas, a saber, educación y enculturación, pueden ocupar posiciones intermedias y periféricas, llegando a todos los niveles de la organización.

La cuarta hipótesis introduce la variable de identidad.

(4) Los discursos de identidad desarrollados dentro de la propia organización tienden a dar estabilidad en dos vertientes: cohesión interna desarrollando una identidad propia de ciertos roles establecidos, muy relacionados con el género y la edad; y frente al exterior, desarrollando un discurso de identidad diferenciada con el resto de la sociedad sin entrar en competencia con el orden establecido en la sociedad donde están inmersos y asimilando algunas de las diferencias de estas últimas.

\section{Métodos y técnicas utilizados}

El estudio se ha basado en una encuesta a voluntarios, destinada a dibujar un esbozo de la red de conocidos de los encuestados. Los puntos clave que

\footnotetext{
${ }^{5}$ Diagnóstico de Marcuse, $H$. recogido en Fernández, Sergio Pablo (1997): "Habermas y la teoría crítica de la sociedad, legado y diferencias de la teoría de la comunicación" Cinta de Moebio no1. Septiembre de 1997. Facultad de Ciencias Sociales de la Universidad de Chile, pág. 1. Disponible en: http://rehue.csociales.uchile.cl/publicaciones/moebio/01/frames30.htm
} 


\section{perifèria \\ Número 2, mayo 2005 \\ www. periferia.name}

he intentado buscar son: grado de centralidad, poder y puntos de división del grupo. Las encuestas se han redactado teniendo en cuenta las variables de edad, educación, sexo, rango dentro de la religión, nivel socio-económico y frecuencia e intensidad de los encuentros.

La recogida y análisis de los datos han sido obtenidos mediante la utilización de los siguientes métodos:

Observación de campo, realizada desde enero de 2004, fecha en que tengo registrado el primer encuentro hasta la actualidad, ya que continúo con la misma. Los lugares escogidos para las observaciones son los centros que la I glesia SUD tiene en Barcelona.

Encuesta rellenada a mano ${ }^{6}$. El encuestado tenía que contestar a 8 preguntas sobre su red personal en Barcelona y enumerar una serie de características de las personas elegidas, a modo de ficha personal, teniendo en cuenta las variables que enumeraré más adelante. Sólo se ha tenido en cuenta en las respuestas las personas que integran las redes personales establecidas en Barcelona y área metropolitana, desestimando cualquier otro conocido o familiar fuera de esta área geográfica. No tuve problema a la hora de que los encuestados aceptasen las variables, sólo algunas protestas por rango bar de la variable LUGARES DE ENCUENTRO, en muchas de las encuestas me encontré tachada esta palabra.

Los programas usados en el análisis de los datos obtenidos por los formularios han sido principalmente UCINET y SPSS. El programa UCINET permite el análisis de redes pudiendo observar así de manera estructural la heterogeneidad del grupo. El programa SPSS permite hacer un esbozo de la población en conjunto, sin tener en cuenta las relaciones entre ellos, y el análisis de redes dibuja el sociograma de los sujetos sociales locales.

Todos los voluntarios son del mismo sexo, misioneros ${ }^{7}$, no autóctonos, viven en Barcelona desde hace 1 año, tienen entre los 19 y 23 años, de

\footnotetext{
${ }^{6}$ La elaboración de la encuesta se ha basado en el libro de Requera Santos, Félix (1996): "Redes sociales y cuestionarios" Ed, CIS en la colección Cuadernos metodológicos, Madrid.

${ }^{7}$ Personas que han viajado desde su lugar de origen y que se dedican
} 


\author{
perifèria \\ Número 2, mayo 2005 \\ www. periferia.name
}

clase socioeconómica que va desde media hasta alta, han finalizado los estudios de secundaria y realizan juntos actividades recreativas de carácter cultural e incluso algunos comparten piso. Los formularios se rellenaron en grupo en uno de los centros que la Iglesia SUD tiene en Barcelona.

Las relaciones que he tenido en cuenta son de socialización ya que no he considerado las relaciones familiares, ni escolares o de trabajo, sólo las establecidas en Barcelona durante el tiempo que ha durado la misión.

\title{
Posición de cercanía y lejanía dentro de las redes personales: la importancia del rol y el estatus
}

Teniendo en cuenta las preferencias de relación de las personas consultadas he obtenido la siguiente gráfica de las relaciones personales.

En lo concerniente a los actores más centrales e influyentes, según las múltiples medidas del grado de centralidad, se tiene en cuenta el grado de salida de los vínculos (si este es alto, se dirá que el actor es influyente). Si el actor social recibe muchos vínculos, se dirá que posee prestigio.

El siguiente cuadro de resultados compara varios resultados sobre las medidas de centralidad, que son Degree para grado de conexiones, closeness para la medida de cercanía, betweenness para la medida intermediación y Eigenvector para encontrar a los actores más centrales. Hay dos grupos de nodos: los que poseen un alto grado de intermediación y los que tienen un grado de intermediación entre 1 y 0 . Los ocho primeros forman parte del primer grupo.

exclusivamente a predicar su religión y enseñar los preceptos básicos durante un periodo de 2 años. 


\section{perifèria}

Número 2, mayo 2005

www. periferia. name

Normalized Centrality Measures

\begin{tabular}{rrrrrr} 
& & 1 & 2 & 3 & 4 \\
& Degree & Closeness & Betweenness & Eigenvector \\
\hline 1 & 1 & 29.310 & 56.863 & 20.882 & 39.018 \\
2 & 2 & 20.690 & 54.206 & 7.683 & 39.356 \\
3 & 3 & 24.138 & 54.206 & 6.468 & 41.014 \\
4 & 4 & 36.207 & 61.053 & 24.440 & 50.211 \\
5 & 5 & 34.483 & 58.586 & 27.562 & 42.450 \\
6 & 6 & 24.138 & 49.153 & 13.463 & 31.633 \\
7 & 7 & 32.759 & 59.794 & 26.413 & 45.524 \\
8 & 8 & 36.207 & 61.053 & 25.511 & 49.929 \\
9 & 9 & 10.345 & 48.333 & 0.052 & 27.490 \\
10 & 10 & 3.448 & 39.189 & 0.000 & 8.449 \\
11 & 11 & 1.724 & 36.478 & 0.000 & 4.047 \\
12 & 12 & 1.724 & 36.478 & 0.000 & 4.047 \\
13 & 13 & 1.724 & 36.478 & 0.000 & 4.047 \\
14 & 14 & 13.793 & 53.704 & 0.934 & 35.173 \\
15 & 15 & 10.345 & 51.327 & 0.000 & 27.639 \\
16 & 16 & 3.448 & 39.189 & 0.000 & 8.449 \\
17 & 17 & 1.724 & 36.478 & 0.000 & 4.047 \\
18 & 18 & 5.172 & 40.845 & 0.000 & 12.850 \\
19 & 19 & 1.724 & 36.478 & 0.000 & 4.047 \\
20 & 20 & 3.448 & 39.189 & 0.000 & 8.449 \\
21 & 21 & 1.724 & 35.366 & 0.000 & 4.082 \\
22 & 22 & 1.724 & 35.366 & 0.000 & 4.082 \\
23 & 23 & 3.448 & 38.411 & 0.000 & 9.461 \\
24 & 24 & 3.448 & 38.411 & 0.000 & 9.461 \\
25 & 25 & 6.897 & 42.336 & 0.000 & 17.921 \\
26 & 26 & 6.897 & 42.336 & 0.000 & 17.921 \\
27 & 27 & 3.448 & 38.411 & 0.000 & 9.461 \\
28 & 28 & 3.448 & 38.411 & 0.000 & 9.461 \\
29 & 29 & 3.448 & 40.559 & 0.000 & 10.386 \\
30 & 30 & 1.724 & 38.158 & 0.000 & 5.208 \\
31 & 31 & 1.724 & 38.158 & 0.000 & 5.208 \\
32 & 32 & 1.724 & 38.158 & 0.000 & 5.208 \\
33 & 33 & 1.724 & 38.158 & 0.000 & 5.208 \\
34 & 34 & 1.724 & 37.179 & 0.000 & 4.403 \\
35 & 35 & 1.724 & 37.179 & 0.000 & 4.403 \\
36 & 36 & 1.724 & 37.179 & 0.000 & 4.403 \\
3737 & 3.448 & 40.278 & 0.308 & 7.683 \\
38 & 38 & 3.448 & 40.845 & 0.000 & 9.124 \\
39 & 39 & 1.724 & 37.179 & 0.000 & 4.403 \\
4040 & 1.724 & 37.179 & 0.000 & 4.403 \\
41 & 41 & 5.172 & 42.647 & 0.308 & 12.862 \\
42 & 42 & 1.724 & 37.179 & 0.000 & 4.403 \\
43 & 43 & 1.724 & 33.143 & 0.000 & 3.281 \\
44 & 44 & 3.448 & 39.456 & 0.000 & 8.459 \\
45 & 45 & 1.724 & 33.143 & 0.000 & 3.281 \\
46 & 46 & 1.724 & 33.143 & 0.000 & 3.281 \\
& & & & &
\end{tabular}




\section{perifèria}

Número 2, mayo 2005

www. periferia.name

$\begin{array}{lllll}4747 & 3.448 & 39.456 & 0.000 & 8.459 \\ 4848 & 1.724 & 37.662 & 0.000 & 4.722 \\ 4949 & 1.724 & 37.662 & 0.000 & 4.722 \\ 5050 & 1.724 & 37.662 & 0.000 & 4.722 \\ 5151 & 1.724 & 37.662 & 0.000 & 4.722 \\ 5252 & 1.724 & 37.662 & 0.000 & 4.722 \\ 5353 & 1.724 & 37.662 & 0.000 & 4.722 \\ 5454 & 1.724 & 37.662 & 0.000 & 4.722 \\ 5555 & 1.724 & 38.158 & 0.000 & 5.178 \\ 5656 & 1.724 & 38.158 & 0.000 & 5.178 \\ 5757 & 1.724 & 38.158 & 0.000 & 5.178 \\ 5858 & 1.724 & 38.158 & 0.000 & 5.178 \\ 5959 & 1.724 & 38.158 & 0.000 & 5.178\end{array}$

DESCRIPTIVE STATISTICS FOR EACH MEASURE

\begin{tabular}{lccccc}
\multicolumn{1}{c}{} & 1 & 2 & 3 & 4 & \\
& & \multicolumn{2}{c}{$\begin{array}{c}\text { Degree } \\
\text { Closeness }\end{array}$} & Betweenness & Eigenvector \\
\hline 1 & Mean & 6.663 & 41.202 & 2.611 & 12.589 \\
2 & Std Dev & 9.662 & 7.238 & 7.157 & 13.435 \\
3 & Sum & 393.103 & 2430.928 & 154.023 & 742.722 \\
4 & Variance & 93.357 & 52.383 & 51.229 & 180.512 \\
5 & SSQ & 8127.229 & 103250.125 & 3424.619 & 20000.000 \\
6 & MCSSQ & 5508.072 & 3090.621 & 3022.532 & 10650.229 \\
7 & Euc Norm & 90.151 & 321.326 & 58.520 & 141.421 \\
8 & Minimum & 1.724 & 33.143 & 0.000 & 3.281 \\
9 & Maximum & 36.207 & 61.053 & 27.562 & 50.211
\end{tabular}

Los actores más influyentes son el no 4, 5, 7 y 8, ya que cuentan con más grados de salida y entrada (degree), esto posibilita que su grado de cercanía con los nodos sea mayor, lo mismo que su grado de intermediación y de centralidad.

Hay que destacar los casos 14 y 15 que cuentan con el mayor número de conexiones, son muy centrales y tienen un alto grado de cercanía al resto de los nodos, pero apenas tienen capacidad de intervención.

Los casos 18, 24, 2529 y 41 tienen similares características aunque no tan acentuadas. La falta de este dato indica que no tienen conexiones de salida, tal como se puede ver en la gráfica de abajo, ya que no estuvieron presentes en la encuesta pero fueron nombrados por todos. 


\section{perifèria \\ Número 2, mayo 2005 \\ www.periferia.name}

Si observamos los datos estadísticos descriptivos para cada medida, se observa una fuerte diferencia entre la media (mean) y la desviación estandar (Std dev), exceptuando en la medida del eigenvector, lo que apunta a una desigualdad estructural y la diferenciación entre actores centrales y periféricos.

No es casualidad que la medida que ubica la dimensión entre las distancias de los actores sea la única que permanece estable. Si se observa la diferencia entre las medidas máximas y mínimas, veremos la fuerte oscilación que apunta a esta afirmación. La media más alta es la de cercanía (closeness) y la que más varía la de intermediación.

Estas medidas apuntan a un gran número de actores sumidero (reciben vínculos) frente a un menor número de actores fuente (emiten vínculos). Esto puede deberse a que las personas nombradas fueron muchas más de las que participaron en la encuesta, ya que cada participante enumera su red personal, que no necesariamente se hallaba presente. Destaca en los estadísticos descriptivos la baja centralidad, causada, a mi modo de ver por el defecto de forma expuesto anteriormente. La fuerte diferencia entre los grados de salida y de entrada en la varianza demuestra que la población es estructuralmente heterogénea ya que hay una fuerte diferencia en ambos casos, siendo mucho mayor el grado de salida que el de llegada.

Si observamos la red veremos que todos los casos donde aparecen grados de salida se sitúan en el centro, exceptuando los casos 14 y 15 que cuentan con gran prestigio, ya que han sido nombrados por todos los encuestados. 


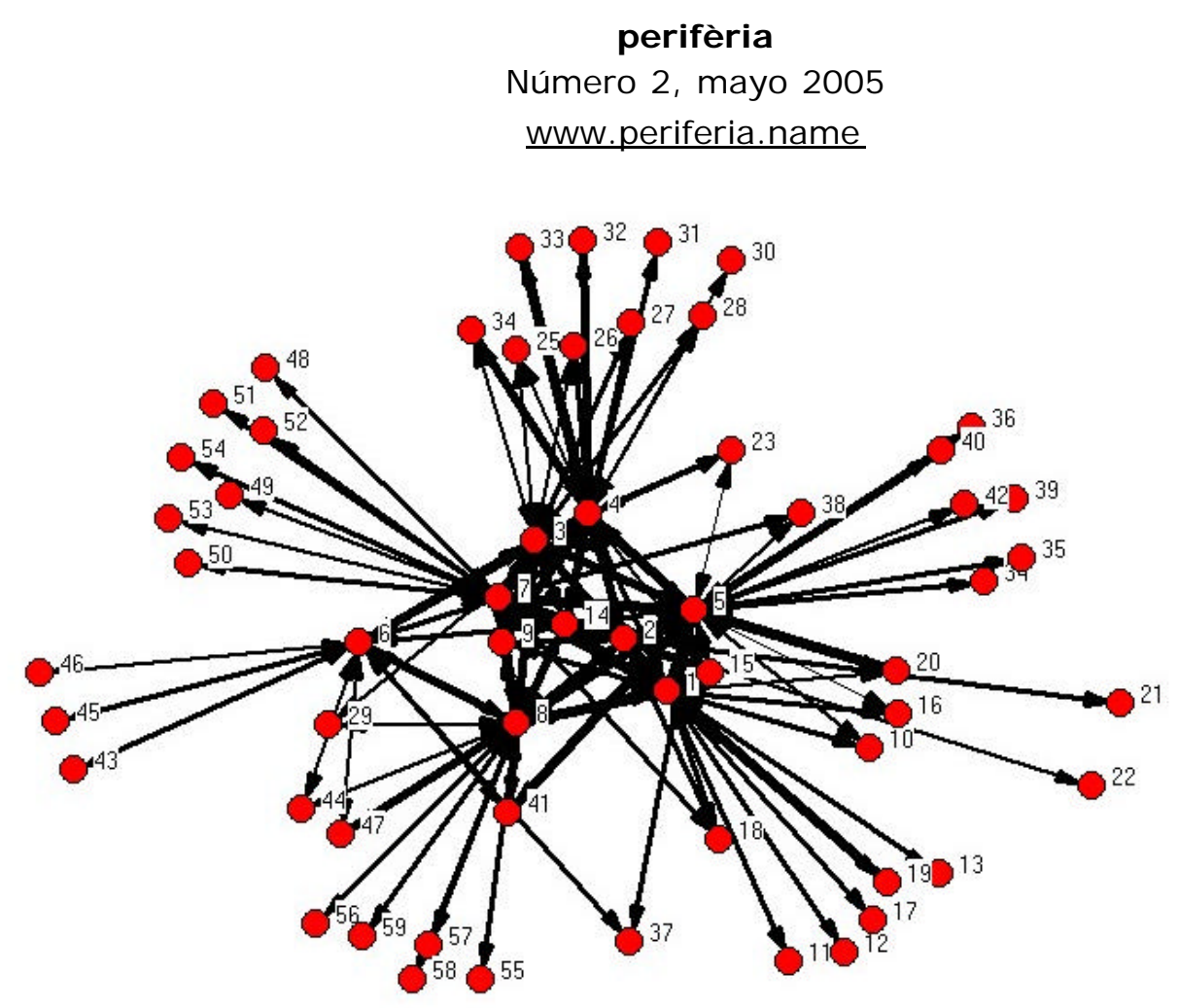

Se puede decir, por tanto, de la red que:

Hay dos partes en la red. Los ocho primeros casos junto con los casos 14 y 15 forman un núcleo duro, cohesionado, tal como se puede ver en el gráfico de la red. La segunda parte estaría formada por los vínculos, establecidos por las relaciones personales, que salen del núcleo hacia la periferia.

Las ramificaciones que salen de los nodos que integran el núcleo duro forman parte de las redes personales de éstos.

El núcleo duro está centrado en el elemento 14, que se sitúa en el epicentro de la red.

Las conexiones entre los nodos del núcleo son orientadas.

La densidad de las conexiones es más fuerte en el núcleo compuesto de los nodos 1 al 9, 14 y 15, y más débil en la periferia. El núcleo duro gira en torno al 14 que se sitúa en el epicentro.

La elaboración de esta red primaria señala la existencia de un círculo más cerrado dentro de las redes personales que tiene expandirse por la periferia a partir de los nodos del núcleo primario.

El núcleo duro tiende a ser homogéneo pero las redes personales que se expanden hacia la periferia procedentes de este núcleo duro son más 


\author{
perifèria \\ Número 2, mayo 2005 \\ www. periferia.name
}

heterogéneas. La existencia de esta diferencia entre los miembros apunta a la aparición de núcleos ego-centrados que podía apuntar a posibles subestructuras. Para ver esta posibilidad he cruzado distintas variables.

\title{
El género y edad: bases de la identidad y su posición en la red
}

La primera clasificación ha sido establecida teniendo en cuenta el sexo y la edad. Estas variables las considero básicas, ya que son comunes para todos los grupos humanos a la hora de establecer los criterios de diferenciación social.

Las personas encuestadas a la hora de establecer las relaciones más directas prefieren relacionarse con personas de su edad y sexo, así si observamos los datos de los estadísticos descriptivos tenemos que la mayoría de las personas nombradas en la encuesta son hombres (79.9\%) y domina la franja de edad comprendida entre los 18 y los 29 años, en un $61 \%$, franja de edad que corresponde a los encuestados.

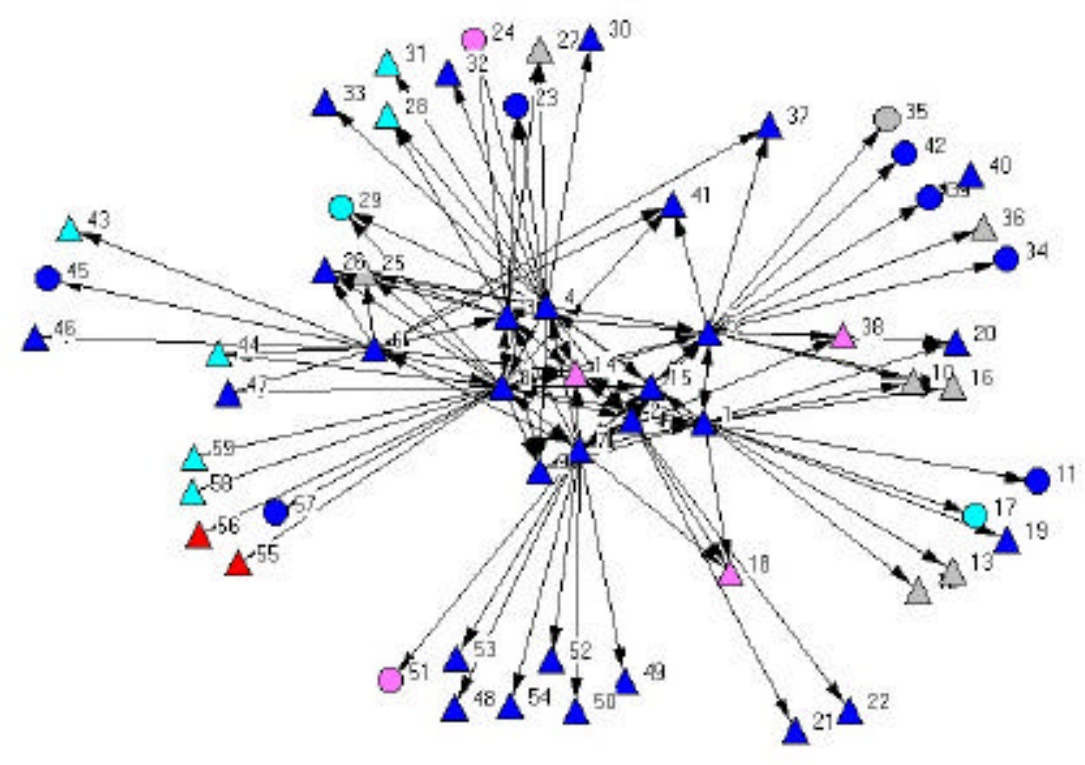

Leyenda:
$\Delta^{\text {Hombre }}$
( Mujer

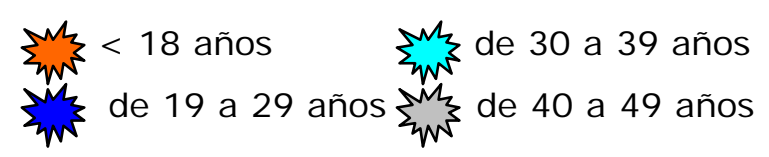
$\sum_{w^{3}}^{m}>50$ años 


\author{
perifèria \\ Número 2, mayo 2005 \\ www. periferia.name
}

El número 14 es el nodo central, a pesar de formar parte del último grupo de edad, su posición indica que posee un fuerte prestigio dentro de las personas del núcleo duro y posee una posición claramente privilegiada. Hay otro criterio a la hora de establecer las relaciones personales dentro del grupo.

\title{
Dos factores importantes en las relaciones: el origen y la edad
}

A pesar de que la mayoría de los encuestados eran de EE.UU. hay un elevado porcentaje de personas procedentes de Latinoamérica, llegando a ser casi la mitad de los nombrados y superando incluso a los estadounidenses. Ambos grupos suman en conjunto un total del 78\%. La población autóctona es la que menos se ha aparecido reflejada en la encuesta y junto con a Asiática y la procedente de Europa del Este (Euro. Est.), cuya presencia es casi simbólica.

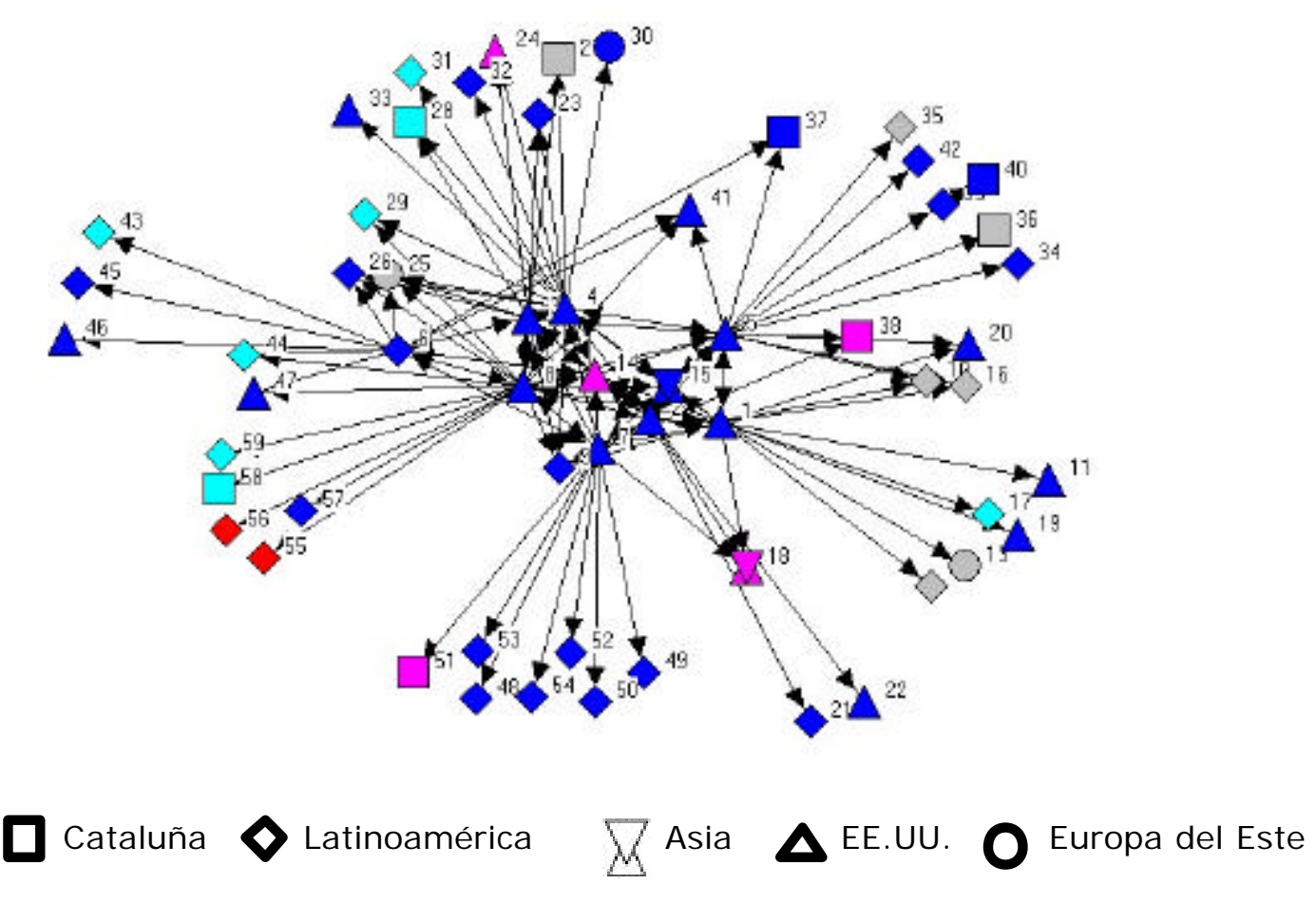




\section{perifèria \\ Número 2, mayo 2005 \\ www.periferia.name}

La edad y el género son importantes, pero no es desdeñable la variable procedencia. Si bien Isidro Maya señala que el sentido de comunidad “(...) se complementa con la percepción de arraigo territorial (...)" parece que el núcleo duro está identificado con la percepción de desarraigo. Todos los integrantes del mismo tienen la característica común de que no son catalanes y la gran mayoría son procedentes de EE.UU., exceptuando uno de Asia y dos de Latinoamérica.

\section{Dos importantes indicadores para poder acceder al saber: estudios y nivel socio-económico}

La mayoría de las personas que aparecen en las fichas han terminado los estudios de secundaria, concretamente el $72.9 \%$ de las personas. Esta variable utilizada en el análisis estadístico descriptivo es la de categoría socioeconómica, clasificada por los propios encuestados. La mayoría de las personas nombradas pertenecen a un estatus medio, más de la mitad, disminuyendo hacia los extremos. También se observa una similitud exacta entre los rangos bajo/medio y medio/alto. Si se tiene en cuenta los niveles medios -bajo/medio, medio, medio-alto- el total llega a ser de un $84.7 \%$ $(23.7+37.3+23.7)$.

Si identificamos a cada persona con una característica, podremos observar donde se sitúan la mayoría y la minoría dentro del grafo de las redes personales.

\footnotetext{
8 Maya Janeiro, Isidro en "Estrategias de promoción: Potenciación comunitaria y Sentido de comunidad" Concurso a una plaza del cuerpo docente de Profesores Titulares de la Universidad del área de Psicología Social, Universidad de Sevilla, 2003. Pág 11.
} 


\section{perifèria}

Número 2, mayo 2005

www. periferia.name

El grafo resultante después de cruzar los datos nivel socio-económico con educación es clarificador si se tienen en cuenta las anteriores variables ya presentadas.

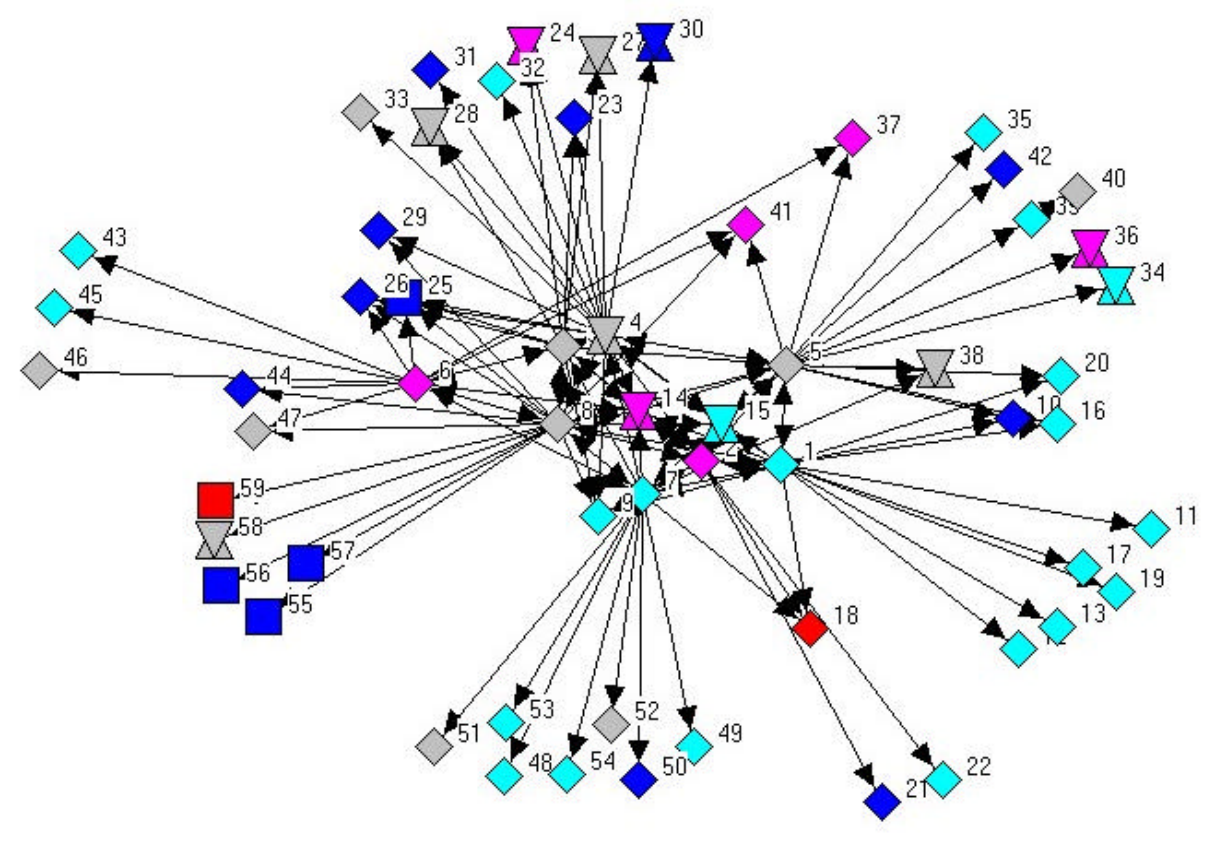

Nivel educativo

$\square$ Primaria $\diamond$ secundaria $\mathbb{B}$ Universidad

Nivel socio-económico

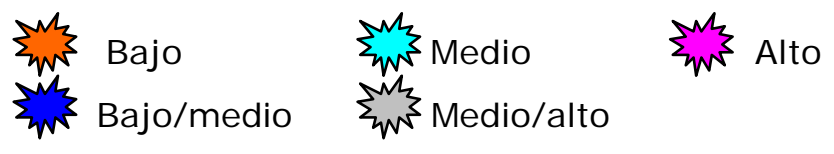

Parece que hay un criterio de selección. Hay sólo dos personas con un nivel socio-económico bajo y no ocupan posiciones centrales, en concreto la que está más alejada del núcleo principal cuenta con estudios primarios ( $n$ 은).

Todos los que cuentan con estudios primarios aparecen en la misma egored, junto con una persona de clase media/alta con estudios universitarios finalizados, y están situados en la periferia. Cabe destacar que dos de estos nodos se encuentran en la franja de menores de edad y es posible que todavía continúen sus estudios. 


\section{perifèria}

Número 2, mayo 2005

www. periferia.name

\section{El género y el rango en las redes personales: estatus y rol}

He de especificar que los rangos diácono, mormones, misioneros y rango específico son todos integrantes de la Iglesia SUD $^{9}$, los que no forman parte de la formación religiosa los he considerado dentro del grupo no Iglesia SUD. La mayoría de las personas nombradas han sido misioneros (22 sobre un total de 59 nodos), y las que menos han sido las personas que no pertenecen a la Iglesia SUD, si bien hay que señalar que estas están estudiando para poder formar parte de la misma. El siguiente grupo numeroso es el de los mormones, son integrantes de la I glesia SUD pero no se ha especificado su rango. Hay otro grupo diferenciado, y minoritario, los diáconos, son jóvenes varones menores de edad.

\section{Religión}

\begin{tabular}{|l|r|r|r|r|}
\hline & Frecuencia & Porcentaje & $\begin{array}{c}\text { Porcentaje } \\
\text { válido }\end{array}$ & $\begin{array}{c}\text { Porcentaje } \\
\text { acumulado }\end{array}$ \\
\hline No Iglesia SUD & 4 & 6,8 & 6,8 & 6,8 \\
Diácono & 3 & 5,1 & 5,1 & 11,9 \\
Mormón & 20 & 33,9 & 33,9 & 45,8 \\
Misionero & 22 & 37,3 & 37,3 & 83,1 \\
Rango específico & 10 & 16,9 & 16,9 & 100,0 \\
Total & 59 & 100,0 & 100,0 & \\
\hline
\end{tabular}

La comunidad es identificada por las relaciones que establecen, el grupo da seguridad, sensación de apoyo mutuo, información y afecto. La presencia de algunas mujeres en las redes personales parecen seguir este patrón, ya que no casi no tienen influencia dentro del grupo. Todas son mormonas y algunas, muy pocas, poseen un cargo dentro de la organización religiosa, bien son misioneras o bien poseen un "rango específico" o rol, pero el estatus no queda reflejado en esta gráfica por la tendencia que se tiene

\footnotetext{
${ }^{9}$ Dentro de este rango considero a las personas que tienen un rol específico diferenciado de los misioneros dentro de la Iglesia SUD.
} 


\section{perifèria \\ Número 2, mayo 2005 \\ www. periferia.name}

dentro del núcleo a preferir a las personas del mismo sexo y procedencia a la hora de establecer las redes personales.

Leyenda:

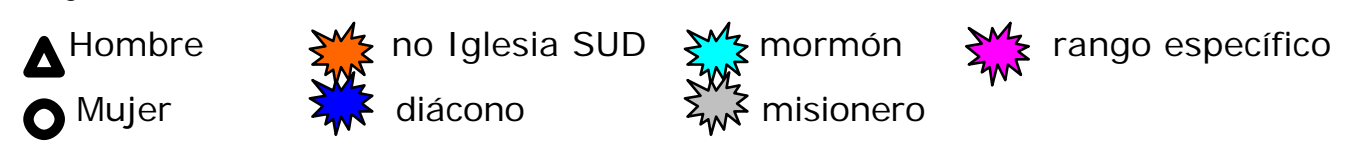

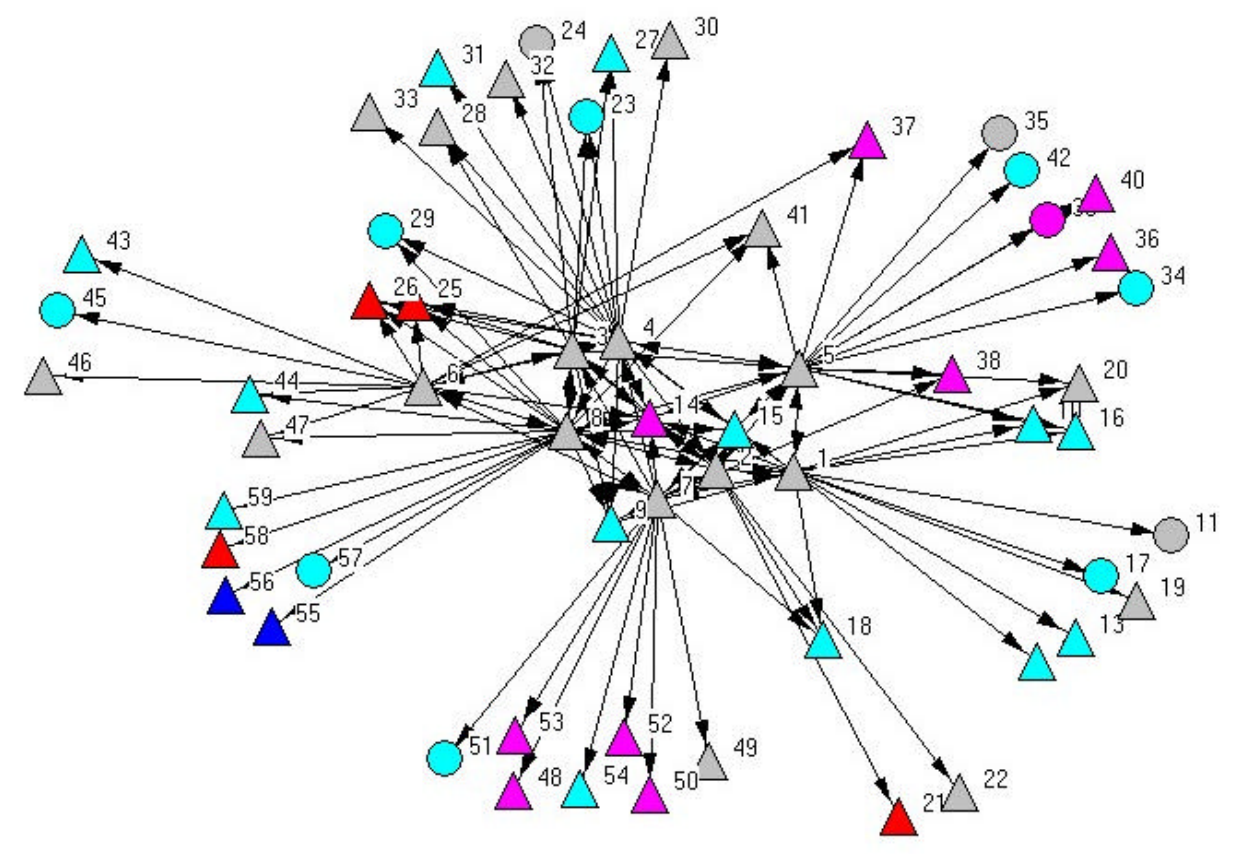

La situación cambia con los hombres. Se entiende la excesiva centralidad del no 14, varón, mayor de 50 años, procedente de EE.UU. y con cargo específico. Tiene un gran prestigio dentro del grupo. Excepto esta persona, el resto de las posiciones de prestigio dentro de la red se reparten entre latinoamericanos y catalanes.

Los datos socio-económicos, junto con la educación, edad y el sexo son relevantes a la hora de ver el status de cada nodo en la egored presentada. Como se ha visto anteriormente, el rol dentro de Iglesia SUD no es relevante a la hora de ocupar una posición central en la egored presentada. Las variables utilizadas a la hora de "personalizar" los nodos y establecer las pautas de conexión del grupo parecen señalar que el núcleo duro es 


\section{perifèria \\ Número 2, mayo 2005 \\ www. periferia.name}

homogéneo en cuanto al sexo y altamente homogéneo (sólo 1 ó 2 nodos no cumplen estos requisitos) en las variables edad, región geográfica de origen, nivel socioeconómico, nivel educativo y rol dentro de la organización religiosa. Hay una "elite" fuertemente cohesionada que se centra en torno a un nodo de elevado rol dentro de la organización y con un estatus reconocido, mientras que los nodos que están situados alrededor actúan como ampliadores de fronteras. Los nodos que no pertenecen a la Iglesia SUD se sitúan en posiciones más alejadas, si bien ningún nodo esta desconectado del núcleo, haciendo que exista comunicación entre todos los integrantes de la red.

\section{El estatus y la intensidad de las relaciones entre los nodos}

Las conexiones entre todos los miembros de la red no son directas, ya que algunos solo reciben conexiones, otros sólo tienen una y pocos emiten más conexiones de las que reciben. La intensidad de las conexiones entre los nodos y su mayor número permiten un mayor acceso a la información y la capacidad de transmitir la misma al resto de la red.

El enfoque $\mathrm{N}$-Clique permite ver "la longitud de la trayectoria permitida para hacer una conexión con todos los miembros ${ }^{\prime 10}$, este enfoque refleja más las redes de solidaridad y accesibilidad al grupo.

59

122 -cliques found.

1: 12345678914152324252627282930313233

2: 12345678914152526294144475556575859

3: 1234567891415183848495051525354

\footnotetext{
10 Robert A. Hanneman "Introducción a los métodos del análisis de redes sociales" Departamento de Sociología de la Universidad de California Riverside. Cap 7, pág. 8. Disponible en: http://revista-redes.rediris.es/webredes/text.htm
} 


\section{perifèria \\ Número 2, mayo 2005 \\ www. periferia.name}
4: 12345678914153841
5: 1234578910141516182038
6: 1234578910141516203841
7: 123457891415182122
8: 12345781014151620343536373839404142
9: 123456781415373841
10: 123456781415252637414447
11: 12457891011121314151617181920
12: 3467814252637414344454647

Hay 12 agrupaciones posibles y en ninguna aparecen todos los miembros. En estas 12 agrupaciones posibles aparecen nombrados los nodos 4, 7, 8 y 14 (en gris) y en 11 de las 12 agrupaciones posibles aparecen los nodos 1 , 2, 3, 5 y 15 (en turquesa). Todos estos nodos forman parte del núcleo duro. Hay que destacar cierta desconexión del nodo 6 que aunque forma parte del núcleo duro sólo aparece en 7 de los 12 cliques posibles. Algunas de las series corresponden con redes personales de las cuales un solo nodo tiene acceso exclusivo. El clique 1 corresponde a la red personal del nodo 4, El clique 2 corresponde a la red personal del nodo 8, El clique 3 corresponde al nodo 7 , El clique 8 corresponde al nodo 5. La poca representatividad del nodo 6 puede deberse a que comparte red personal con más de 1 nodo y no tiene una red personal en exclusividad.

El análisis se ha centrado desde un punto de vista desde el núcleo hacia la periferia. El rol de los misioneros es ampliar la red del grupo consiguiendo nuevos miembros para el grupo. No quiere decir esto que las personas que aparecen en la red sean futuros miembros del grupo, ya que no se especificaba en la encuesta.

Destaca el escaso número de personas no integrantes de la Iglesia SUD dentro de las redes personales, ya que sólo han aparecido 4 nodos, el 25 , 26, 58 y 21 . Los nodos 25 y 26 se acercan más a este núcleo por su presencia en dos redes personales (nodos 4 y 3), mientras que el 58 (nodo 8) y el 21 (nodo 2) aparecen nombrados en una red personal. La distancia muestra las veces que se ha nombrado a una persona por un determinado 


\section{perifèria \\ Número 2, mayo 2005 \\ www. periferia.name}

número de personas pero no refleja la frecuencia de los encuentros dentro de la red personal.

\section{Conclusiones}

El núcleo duro es fuertemente endógeno, prefieren relacionarse con las personas del mismo sexo, parecido rol y status y misma nacionalidad en sus relaciones más personales aunque expanden la red hacia la periferia que es más heterogénea.

Hay un nodo que cuenta con un gran prestigio. Las características de éste son: varón, mayor de 50 años, educación universitaria, alto estatus económico, posee un rol de reconocido dentro de la organización y procede de EE.UU.

Es un grupo fuertemente patriarcal, las mujeres están relegadas a la periferia independientemente de la cultura, rol o estatus económico del que disponen.

Las 4 personas que no pertenecen a la Iglesia SUD se sitúan en la periferia pero la frecuencia de tiempo y lugares de los encuentros puede ser mayores que los del resto de la periferia, sólo dos de los nodos que se encuentran más cerca del núcleo han visto disminuida la presión del grupo. Estas personas cuentan con más posibilidades de integrarse dentro del mismo. El grupo tiende a ampliarse de forma heterogénea y a abarcar un máximo de posibilidades socioeconómicas y de nivel educativo, aunque estas personas cuentan con una característica común: son todos hombres.

El nivel socioeconómico, el sexo y el nivel educativo tiene importancia a la hora de desempeñar un rol y ocupar un determinado status dentro del grupo. Los nodos centrales son, como mínimo, de clase media, han terminado los estudios de secundaria, son misioneros y varones. 


\section{perifèria}

Número 2, mayo 2005

www. periferia.name

\section{Líneas futuras de investigación}

Las investigaciones posteriores se centrarán en el siguientes puntos:

El rol y el estatus que tienen las mujeres dentro de la organización. Es posible que éstas tengan una importante labor como educadoras y enculturadoras del sistema social, menos centrales que las de obispo o presidente, cargos más administrativos. Por tanto las futuras líneas de investigación irán destinadas a la elaboración de una sociored de la organización, las redes establecidas entre las mujeres, en qué puntos se relacionan con las de los hombres y cómo se establecen los canales educativos y de información.

El modelo de integración que sigue el grupo se acerca más al multiculturalismo que a la asimilación, siguiendo la clasificación de Kymlicka $(2000)^{11}$. En esta última clasificación se conservan las diferencias culturales y de género, este último construido como identidad propia. Esta separación queda reflejada de un modo estructural como mosaico cultural.

Otra interesante línea de investigación es la influencia del origen geográfico a la hora de establecer las relaciones de grupo. Sería interesante observar si los mormones catalanes establecen relaciones dentro y fuera de la organización religiosa y los mormones inmigrados establecen las relaciones mayoritariamente dentro de la organización teniendo a concentrarse por origen geográfico.

\footnotetext{
${ }^{11}$ Recogida por Rodríguez García, Dan (2004) en “Inmigración y mestizaje hoy. Formación de matrimonios mixtos y familias transnacionales en Cataluña", Barcelona: Servei de publicacions de la UAB. Cap. 1.8.
} 


\author{
perifèria \\ Número 2, mayo 2005 \\ www. periferia.name
}

\title{
Agradecimientos
}

Quisiera agradecer a José Luis Molina, doctor en antropología por la UAB y coordinador del doctorado, las correcciones y sugerencias del presente estudio. Muchas gracias, también, a las personas anónimas que se han prestado voluntarias a la encuesta por la paciencia y el tiempo dedicado.

\section{Bibliografía}

Aguilar, Claudia y Molina, José Luis (2004): “Identidad étnica y redes personales entre los jóvenes de Sarajevo" Departamento de Antropología Social y Cultural, Universidad Autónoma de Barcelona.

http://revista-redes.rediris.es/webredes/ivmesahis/identidad.pdf

Fernández, Sergio Pablo (1997): “Habermas y la teoría crítica de la sociedad, legado y diferencias de la teoría de la comunicación" Cinta de Moebio no1. Septiembre de 1997. Facultad de Ciencias Sociales de la Universidad de Chile. Disponible en:

http://rehue.csociales.uchile.cl/publicaciones/moebio/01/frames30.htm

Horowitz, Irving, L. (1963): “Poder, política y pueblo” Editado por el Fondo de Cultura Económica, México-Buenos Aires.

Marcuse, H. (1969): “Industrialización y capitalismo en Max Weber" en "La sociedad industrial y el marxismo" Editorial Quintaria, Buenos Aires, 1969.

Maya Janeiro, Isidro (2003) en “Estrategias de promoción: potenciación comunitaria y sentido de comunidad" Concurso a una plaza del cuerpo docente de Profesores Titulares de la Universidad del área de Psicología Social, Universidad de Sevilla.

Requera Santos, Félix (1996): “Redes sociales y cuestionarios" Ed, CIS en la colección Cuadernos metodológicos, Madrid.

Robert A. Hanneman (2000) “Introducción a los métodos del análisis de redes sociales" Departamento de Sociología de la Universidad de California Riverside. 


\section{perifèria \\ Número 2, mayo 2005 \\ www. periferia.name}

Disponible en: http://revista-redes.rediris.es/webredes/text.htm

Rodríguez García, Dan (2004): “Inmigración y mestizaje hoy. Formación de

matrimonios mixtos y familias transnacionales en Cataluña", Barcelona:

Servei de publicacions de la UAB. 